\title{
A Study on Shifting Shock of Multi-step Electromagnetic Transmission in Interior Permanent Magnet Synchronous Motor for EV
}

\author{
Hong-Sik Lim, Byeong-Chul Lee, Ki-Chan Kim* \\ Department of Electrical Engineering, Hanbat National University, South Korea
}

Copyright $\mathrm{C} 2019$ by authors, all rights reserved. Authors agree that this article remains permanently open access under the terms of the Creative Commons Attribution License 4.0 International License

\begin{abstract}
Background/ Objectives: Apply the electromagnetic multi-step transmission method to expand the average efficiency area of electric vehicles. In this paper, analyze the influence of torque caused by shift shock due to electromagnetic multi-shift. Methods/ Statistical analysis: Research has been conducted to extend the average efficiency range of the motor by applying the electromagnetic multi-step transmission method. In addition, we analyze the effects of shift shocks on the torque of the motor through finite element analysis methods(FEM) analysis. Findings: In this paper, we study the characteristic analysis of the electromagnetic multi-step transmission method of interior permanent magnet synchronous motor. In addition, we analyzed the influence of the shift shock due to the electromagnetic multi-shift on the torque. It has been confirmed that the average efficiency section of the motor is widened by the electromagnetic multi-step transmission method, and it has been confirmed that the difference in the voltage in the shift section adversely affects the torque of the motor. It has been confirmed that the influence on the torque is different based on the shift point. Improvements/ Applications: Analyzes and derives attributes thought FEM analysis using voltage source analysis and current source analysis.
\end{abstract}

Keywords Shifting Shock, IPMSM, Electromagnetic Multi-step Transmission Method, Efficiency Map, FEM

\section{Introduction}

We are interested in the development of environmentally friendly electrical devices due to environmental issues such as global warming worldwide. In particular, development of environmentally friendly vehicles and research on alternative energy, such as electric vehicles, are in the spotlight. As described above, research is being conducted on electrical equipment systems using environmentally friendly electrical energy in equipment using engines. A permanent magnet synchronous motor is often used as the drive acceptance motor of the electric vehicle. In the interior permanent magnet synchronous motor, a rare earth permanent magnet is inserted to a rotor. It has the advantages of high efficiency and high power density, high mechanical strength, and about flux weakening control advantages. In addition, reluctance torque is generated due to the difference between $\mathrm{d}$-axis inductance and q-axis inductance, and magnetic torque and reluctance torque are simultaneously used. In the case of an electric vehicle, the driving range is of the utmost importance, which relates to the efficiency of the used motor of the electric vehicle. In order to extend the driving range by an electric vehicle, it is important not to increase the maximum efficiency but to extend the range of high efficiency. In order to extend the range of high efficiency, in this paper, we applied an electromagnetic multi-step transmission method. In addition, a shift point is generated by the electromagnetic multi-step transmission method. In the case of shift points, the winding specifications are changed, so that the voltage levels are different, which affects the torque.

In this paper, the finite element method is used to extend the high efficiency range of the motor to which the electromagnetic multi-step transmission method is applied, and the influence on the torque by the shift shock due to the difference in voltage level of shift points is analyzed.

\section{Analysis Model and Method}

Figure 1 shows the 2D FEM analysis model of IPMSM basic model with 48 slots of 8 poles and maximum output of $80 \mathrm{~kW}$. In the case of base model, a high efficiency range appears at the high speed operating range, so it is not suitable for the electromagnetic changeover system. Therefore, when applying the electromagnetic conversion 
system thought complementary design, the range of average efficiency can be expanded $[7,8]$.
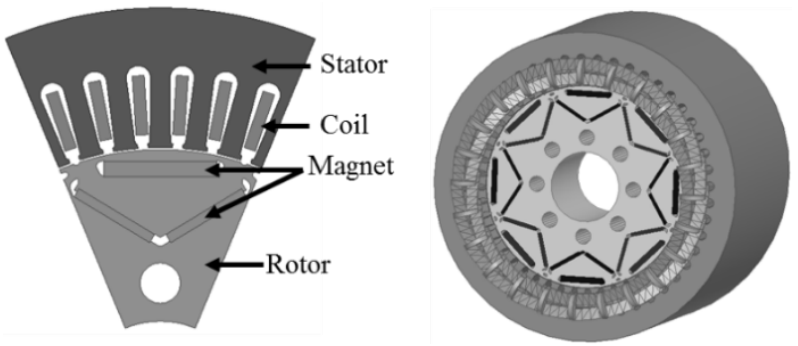

Figure 1. 2D\&3D FEM analysis model

Figure 2 shows a speed torque curve of 1 stage of two parallel branches and 2 stages of four parallel branches when applying the multistage transmission method. By using the electromagnetic switching, high torque can be outputted for 1 stage, but voltage saturation is advanced at low speed and the maximum efficiency range is shown at low speed. In 2 stages Low torque is generated, but resistance decreases, voltage limitation margin occurs, voltage saturation time is slow, it is possible to operate in a wide range at high speed and expand the range of efficiency. Therefore, we have changed the stator winding at $6,000 \mathrm{rpm}$, so that the maximum efficiency section can be used more widely. Figure 3 shows the efficiency map when the changeover is applied to the proposed model. A speed of shifting is selected at which the efficiency is reduced due to weak field control at about $6,000 \mathrm{rpm}$. As can be in the figure 3, the high efficiency area of both methods is greatly expanded at high speed. When the changeover of the stator winding is applied, the average efficiency of the motor increases [11].

In figure 4, the first and second steps are waveforms of voltage the current and when the torque is $127 \mathrm{Nm}$ at the speed change section of $6,000 \mathrm{rpm}$. The current in figure 4 (a) is 246.5 [Arms], the voltage is 127.2 [Vrms], the current in figure 4 (b) is 302.3 [Arms] and the voltage is 92.3 [Vrms]. Since the number of parallel branches is changed and the resistance of the motor changes, the same torque is generated, but the values of current and voltage have different values. Although the number of parallel branches is changed and the voltage and current in the 1st stage mode and the 2 stage mode are different, as shown in Figure 5, it can be confirmed that the $127 \mathrm{Nm}$ same torque is generated[14].

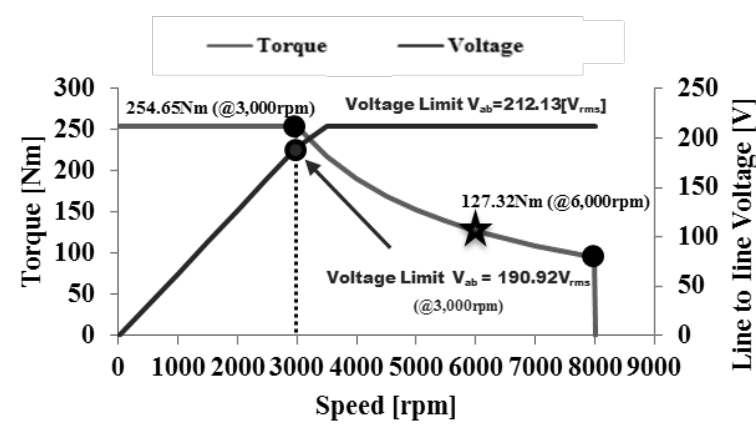

Two parallel branches

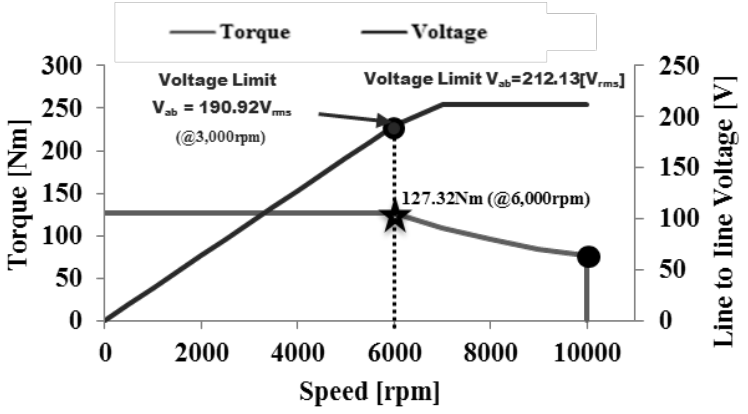

(b) Four parallel branches

Figure 2. Speed-torque curve

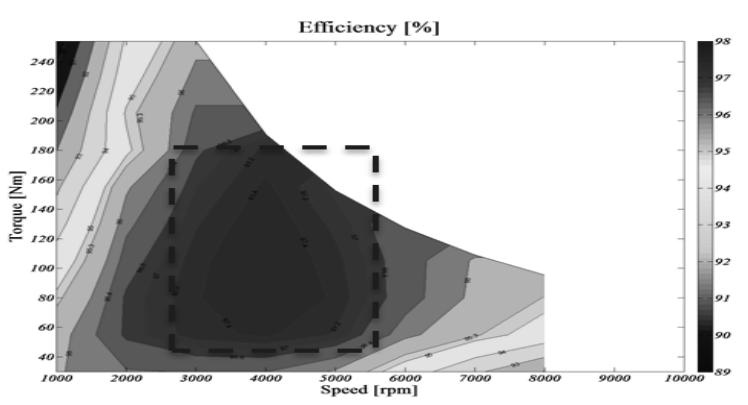

Two parallel branches

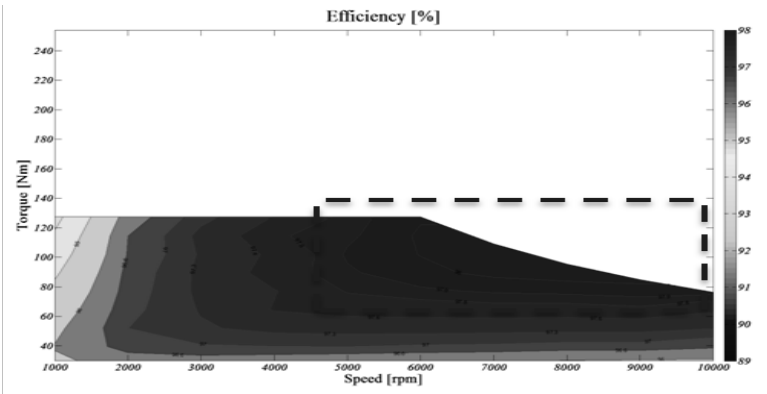

(b) Four parallel branches

Figure 3. Efficiency map 

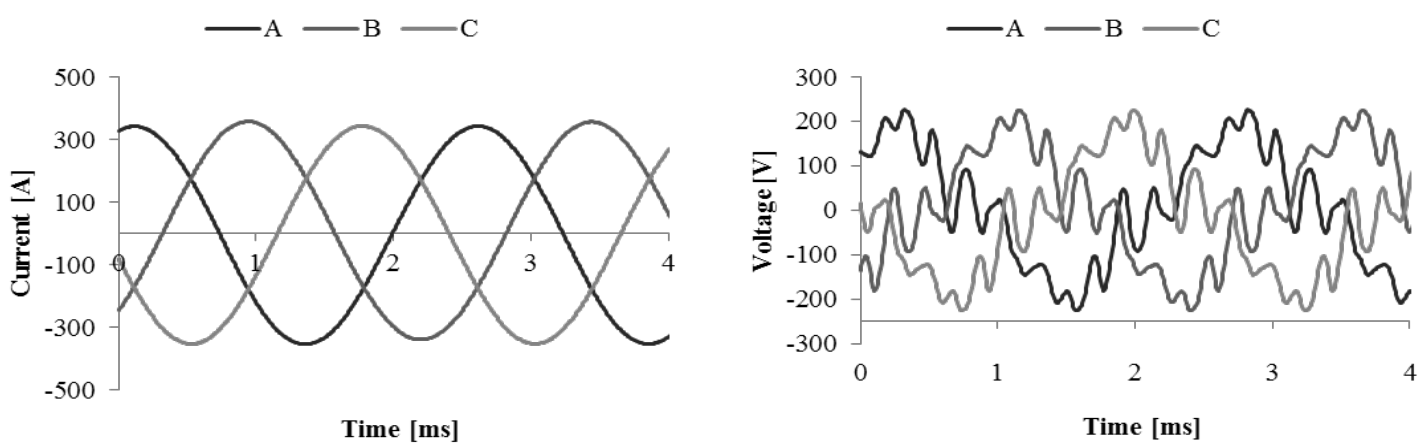

(a) Two parallel branches

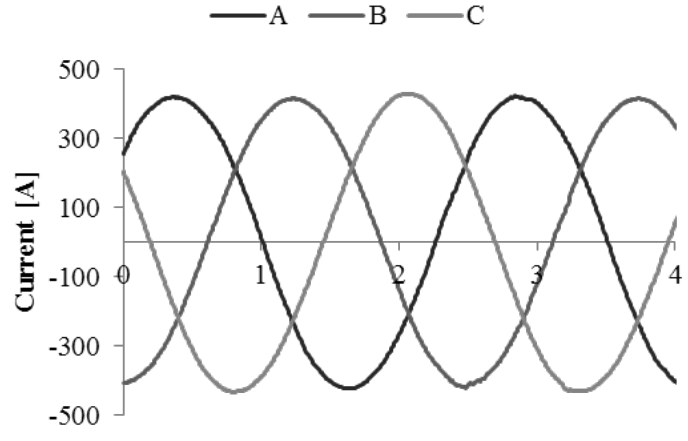

Time $[\mathrm{ms}]$

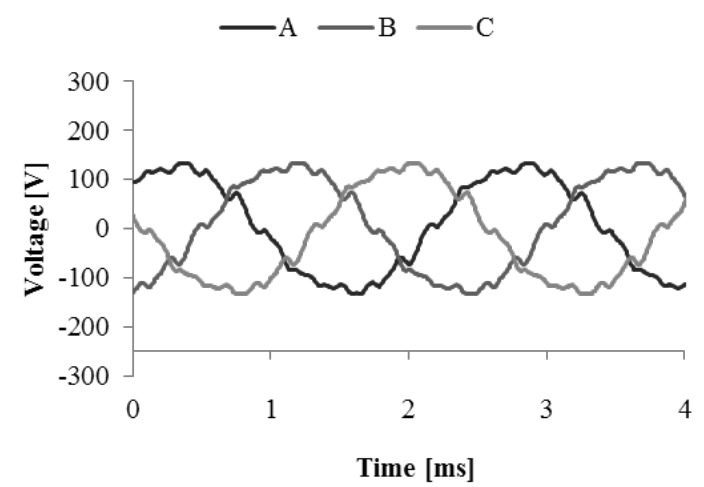

(b)Four parallel branches

Figure 4. Input current and voltage

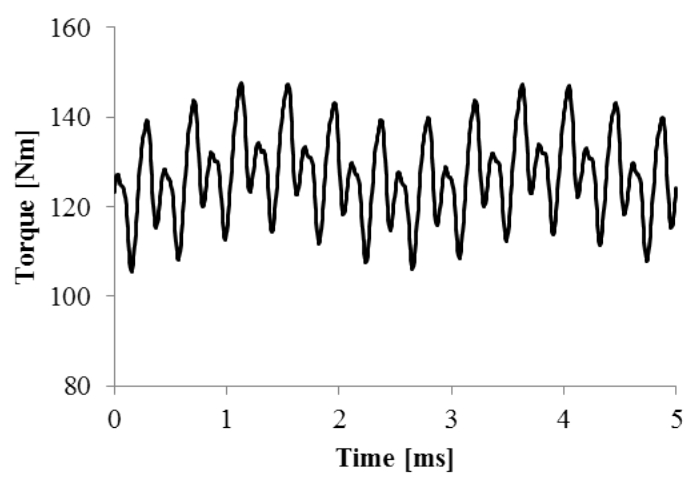

(a) two parallel branches

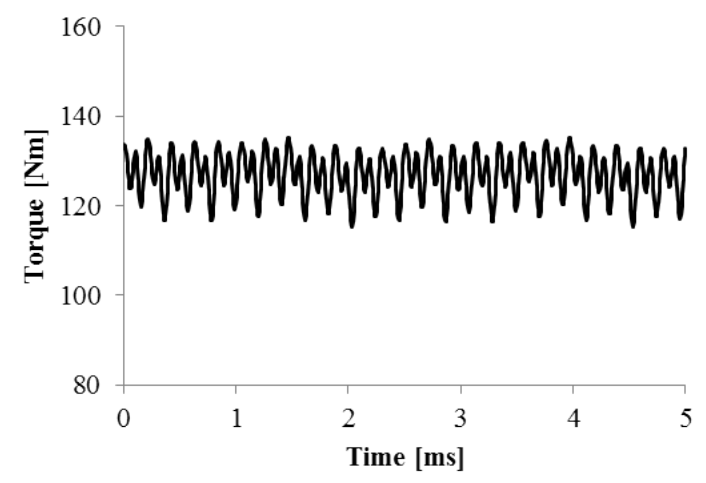

(b) four parallel branches

Figure 5. Torque curve

\section{Electromagnetic Multi-step Transmission Method of Stator Winding}

Figure 6 shows a circuit diagram of the number of parallel branches changeover of the stator winding. As shown in the Figure 6 thyristors are used to control the current flow. At low speed, the drive starts with two parallel branches. In the case of two parallel branches, the current flow through the electromagnetic switching is shown in the 1-step mode. At high speed, the stator winding are converted into four parallel branches. In the case of four parallel branches, the current flows as shown in the 2-step mode.

\section{Shifting Shock of Multi-step Transmission in IPMSM}

When multi-step transmission, change the voltage with reference to the $\mathrm{A}$ phase voltage at 0 degree, 45 degrees, 90 degrees, and all the $\mathrm{A}, \mathrm{B}, \mathrm{C}$ phase voltages were 
zero-crossed. Figure 7 shows voltage waveforms when changing the multi-step transmission described above. Figure 8 shows Currents waveform during changing the multi-step transmission. Depending on the changing the multi-step transmission, the voltage and phase of each phase are different, so the current shows different values for each condition $[9,10,13]$.

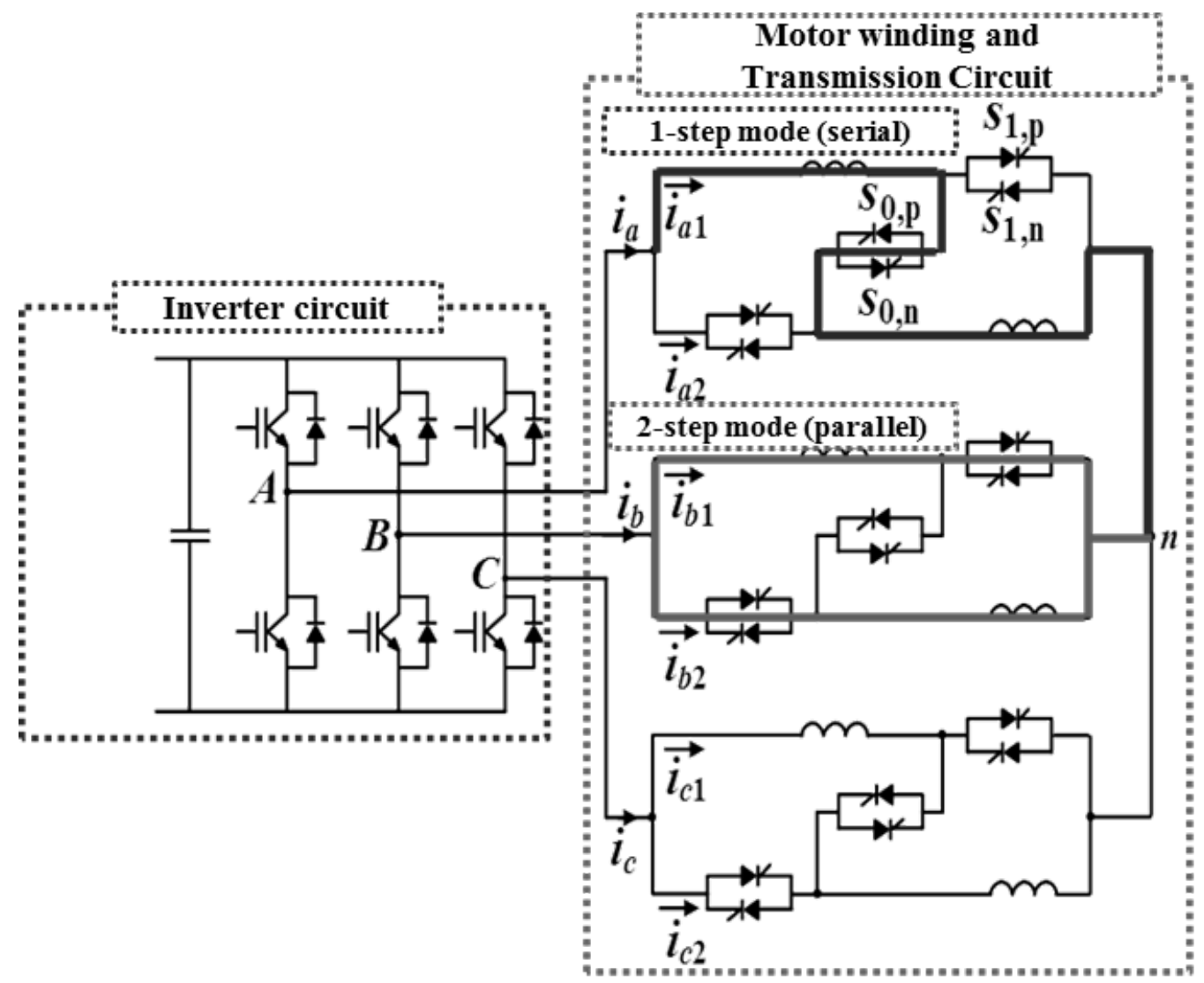

Figure 6. number of parallel branches changeover circuit diagram
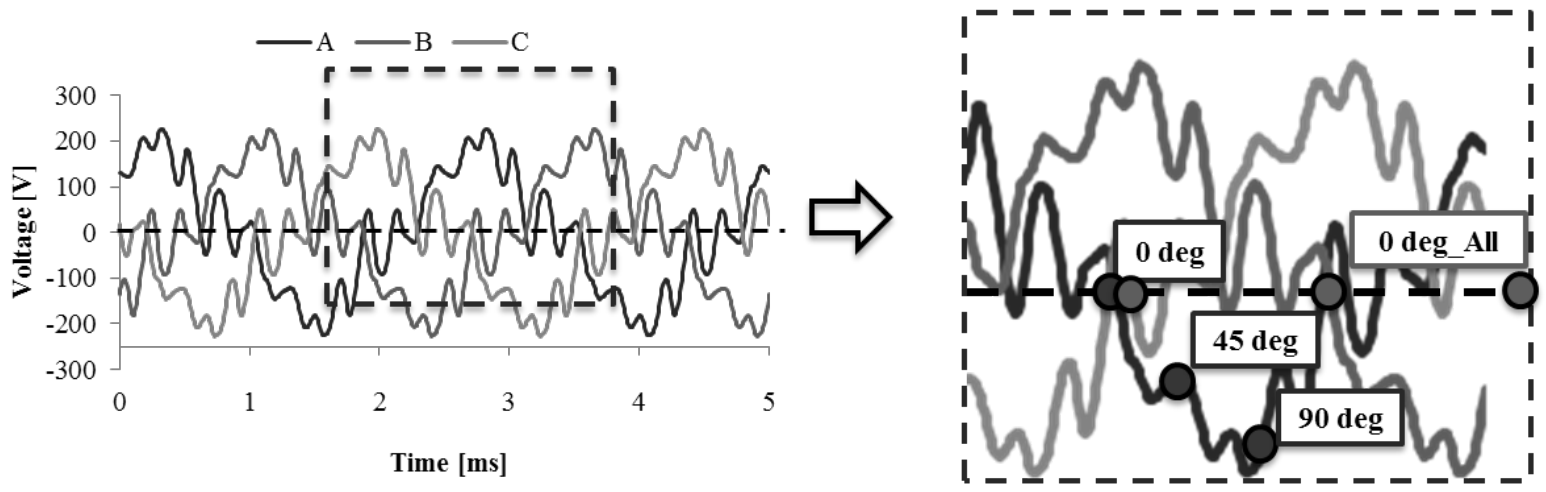

Figure 7. When changing the multi-step transmission voltage waveform. 


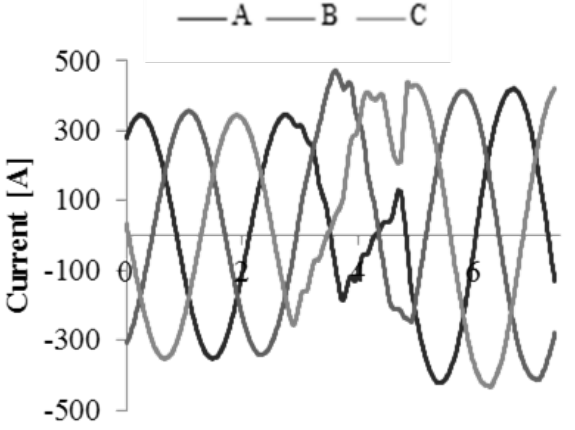

Time [ms]

(a) all the A, B, C phase voltages were zero-crossed
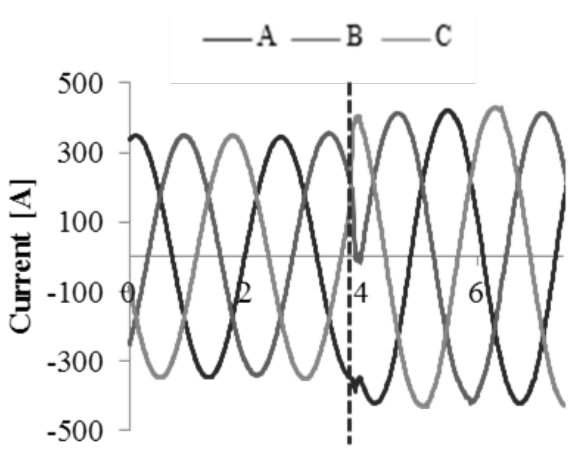

Time [ms]

(c) 45 degrees

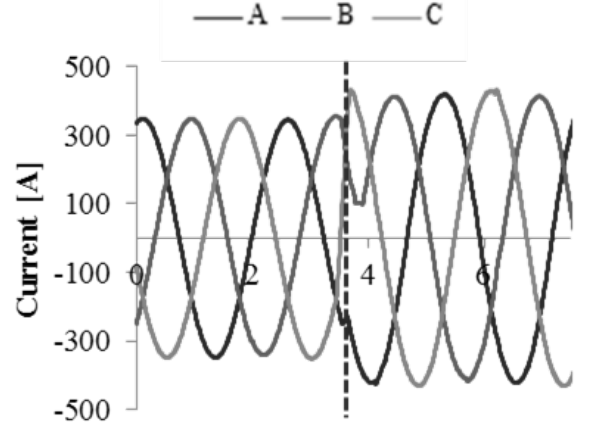

Time [ms]

(b) 0 degrees

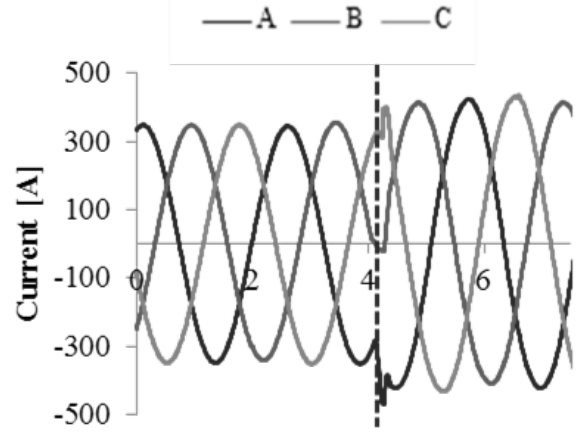

Time [ms]

Figure 8. Current waveform during changing the number of parallel branches

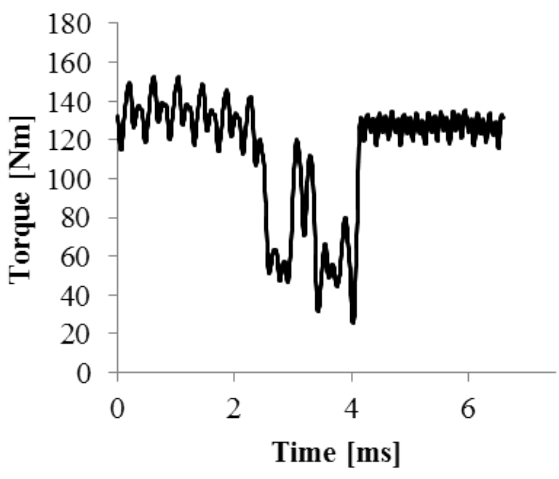

(a) All the A, B, C phase voltages were zero-crossed

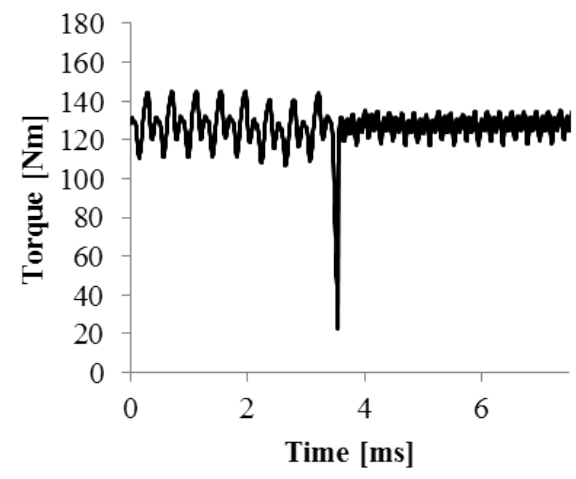

(b) 0 degrees

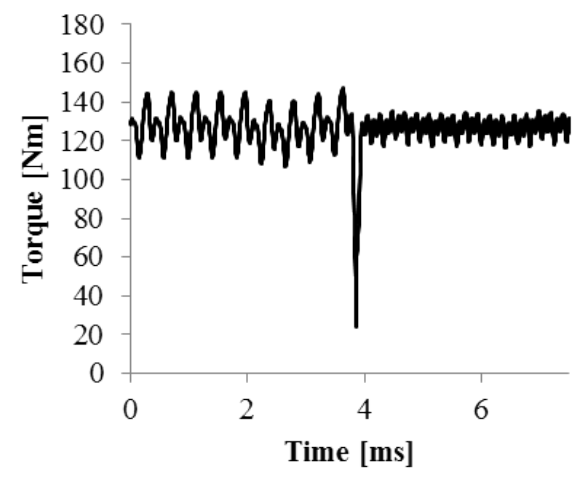

(c) 45 degrees

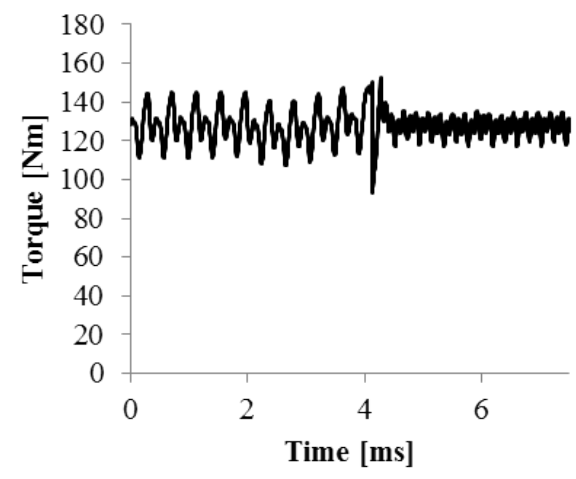

(d) 90 degrees

Figure 9. Torque waveform during changing the number of parallel branches 
Figure 9 is torque waveform displayed when the current waveform of figure 8 is inputted. Depending on the timing of the multi-step transmission, the effect on torque is displayed differently. It has the worst influence on the torque when changing the $\mathrm{A}, \mathrm{B}, \mathrm{C}$ phases by zero crossing. It can be confirmed that when the electrical angle is 90 degrees on the basis of the A phase, the most stable changing is made.

\section{Conclusions}

In this paper, we study shifting shock of IPMSM by multi-step transmission. We designed to the fluctuation of the efficiency map point of the motor of multi-step transmission. In the case of multi-step transmission application, when the maximum efficiency point is 1-step, it is located at low speed and 2-step it is located at high speed. Therefore, the maximum efficiency section is widened[12]. In addition, the method of changing the number of parallel branches, which is a multistep transmission system, was explained. When changing is performed in the multi-step transmission, since the magnitudes and phases of the voltages of the first and second stages are different from each other, the current also changes suddenly. Torque will also be subject to shift shock. The influence on the torque appears differently depending on the timing of changing the number of parallel branches, it was the most reliable when it was 90 degrees.

\section{Acknowledgements}

This work was supported by the Korea Institute of Energy Technology Evaluation and Planning (KETEP) and the Ministry of Trade, Industry \& Energy (MOTIE) of the Republic of Korea (NO.20184030201900).

\section{REFERENCES}

[1] Feng-Sheng Hsu, Min-Fu Hsieh, David G. Dorr-ell, Winding Changeover Permanent-Magnet Generat-ors for Renewable Energy Applications, IEEE TRANSACTIONS ON MAGNETICS, 2012. 48(11): pp. 4168-4171.

[2] Jian Li, Yun hyun Cho, Da woon Choi, Analysis of Rotor Eccentricity in Switched Reluctance Motor With Parallel Winding Using FEM, IEEE TRANSACTIONS ON MAGNETICS, 2009. 45(6): pp. 2851-2854.

[3] Sang-Hyeok Seo, Gyeong-Jae Park, Jung-Min Mun, Yong-Jae Kim, Dae-Woo Km, Sang-Yong Jung, Design Characteristics of IPMSM With Wide Constant Power Speed Range for EV Traction, IEEE TRANSACTIONS ON MAGNETICS, 2017. 53(6), 8105104.

[4] Ho-Chang Jung, Sang-Yong Jung, Deok-Jin Kim, Gyeong-Jae Park, Optimal Design and Validation of IPMSM for Maximum Efficiency Distribution Compatible to Energy Consumption Areas of HD-EV, IEEE TRANSACTIONS ON MAGNETICS, 2017. 53(6), 8201904

[5] Liang Fang, Jung-Ho, Jung-Pyo Hong, Jae-woo Jung, Study on High-Efficiency Performance in Interior Permanent-Magnet Synchronous Motor With Double-Layer PM Design, IEEE TRANSACTIONS ON MAGNETICS, 2008.44(11): pp. 4393-4396

[6] Geun-Ho Lee, Ji-Hyung Bahn, Sung-Il Kim, Jung-Pyo Hong, Torque Ripple Reduction of Interior Permanent Magnet Synchronous Motor Using Harmonic Injected Current., IEEE TRANSACTIONS ON MAGNETICS, 2008. 44(6): pp. 1582-1585.

[7] Ryosuke Akaki , Yasuhito Takahashi , Koji Fujiwara , Makoto Matsushita, Norio Takahashi , Masatsugu Morita, Effect of Magnetic Property in Bridge Area of IPM Motors on Torque Characteristics, IEEE Transactions on Magnetics, 2013, 49 (5).

[8] H. W. Lee, K. D. Lee, W. H. Kim, I. S. Jang, M. J. Kim, J. J. Lee, J. Lee, Parameter design of IPMSM with concentrated winding considering partial magnetic saturation, IEEE Transactions on Magnetics, 2011, 10 (10), pp. 3653-3656.

[9] Liang Chen, David Hopkinson, Jiabin Wang, Andrew Cockburn, Martin Sparkes, William O'Neill, Reduced Dysprosium Permanent Magnets and Their Applications in Electric Vehicle Traction Motors, IEEE Transactions on Magnetics, 2015, 51 (11).

[10] Zhang Qianfan, Cheng Shukang, Song Liwei, Pei, Axial excited hybrid reluctant motor applied in electric vehicles and research of its axial coil signal, IEEE Transactions on Magnetics, 2005, 41 (1), pp. 518-521

[11] Aliyev, A. G., \& Shahverdiyeva, R. O. Development Problems of Information Provision on the Management of High Technology Park. Journal of Information, 2017, 3(1), pp. $1-10$

[12] Amini, M., Alinezhad, A., \& Najafabadiha, H. Ranking Iranian Accredited Laboratories with Proficiency Tests Using Promethee Method. Review of Industrial Engineering Letters, 2014, 1(1), pp. 25-35

[13] Boyinbode, O. Smart Campus: An Implementation of a Cloud-Based Mobile Learning Application. Journal of Information, 2018, 4(2), pp.24-33

[14] Carmen, J., Gutierrez, R., Armando, J., Rosas, P., Torres, G., \& Ulises, P. Utilization of Fish and Mango Wastes on Biological Silage Production. Current Research in Agricultural Sciences, 2018, 5(1), pp.6-14. 